\title{
HICOLM: High-Performance Platform of Large Systems by Using Low Computational Cost Methods
}

\author{
HICOLM: Plataforma de alto desempenho de sistemas grandes usando métodos de \\ baixo custo computacional
}

\author{
Flaviano Williams Fernandes ${ }^{1 *}$
}

\begin{abstract}
For decades, computational simulation models have been used by scientists in search for new materials with technological applications in several areas of knowledge. For this, software based on several theoretical-computational models were developed in order to obtain an analysis of the physical properties at atomic levels. The objective of this work is proposing a widely functional software to analyze the physical properties of nanostructures based on carbon and condensed systems using theories of low computational cost. Therefore, a Fortran language computational program called HICOLM was developed, whose theoretical bases are based on two commonly known models (Tight-binding and Molecular Dynamics). The physical properties of condensed systems can be obtained in the thermodynamic equilibrium in several statistical ensembles, and possible to obtain an analysis of the properties of the material and its evolution in the time-dependent on its thermodynamic conditions like temperature and pressure. Moreover, from the tight-binding model, the HICOLM program is also capable of performing a physical analysis of carbon-based nanostructures from the calculation of the material band structure.
\end{abstract}

Keywords: Tight-binding - Molecular dynamics — nanotechnology — molecular structure

Resumo: Durante décadas, métodos de simulação computacional vêm sendo empregados por cientistas na busca de novos materiais com aplicações de cunho tecnológico em diversas áreas do conhecimento. Para isso, softwares baseados em diversos modelos teóricos-computacionais foram desenvolvidos afim de obter uma análise das propriedades físicas a nível atômico. O objetivo desse trabalho é o desenvolvimento de um software amplamente funcional afim de analisar as propriedades físicas de nanoestruturas baseadas em carbono e sistemas condensados utilizando teorias de baixo custo computacional. Portanto, foi desenvolvido um programa computacional em linguagem Fortran chamado HICOLM, cujas bases teóricas baseiam-se em dois modelos comumente conhecidos (Tight-binding e Dinâmica Molecular). As propriedades físicas de sistemas condensados poderão ser obtidas no equilíbrio termodinâmico em diversos ensembles estatísticos, sendo possível a obtenção de uma análise das propriedades do material e sua evolução no tempo dependente de suas condições termodinâmicas como temperatura e pressão. Além do mais, à partir do método tight-binding, 0 programa HICOLM também é capaz de realizar uma análise física de nanoestruturas baseadas em carbono a partir do cálculo da estrutura de bandas do material.

Palavras-Chave: Tigh-binding - Dinâmica molecular — nanotecnologia - estrutura molecular

${ }^{1}$ Instituto Federal do Paraná, Campus Irati, Irati, Paraná, Brazil

*Corresponding author: flaviano.fernandes@ifpr.edu.br

DOI: http://dx.doi.org/10.22456/2175-2745.92486 • Received: 14/05/2019 • Accepted: 02/07/2019

CC BY-NC-ND 4.0 - This work is licensed under a Creative Commons Attribution-NonCommercial-NoDerivatives 4.0 International License.

\section{Introduction}

In the last decades, the study of nanostructured materials based on carbon has become a target of great interest for researchers and engineers. The theoretical and experimental investigation of its electronic and structural properties has demonstrated that these materials have great applications in several areas of technology, such as in health, engineering, biotechnology and quantum computing. From the theoretical point of view, researchers have been using the most varied computational simulation techniques to analyze electronic and magnetic behavior of several materials. Due to this, graphene has been the subject of great interest by the scientific community due to its physical properties, such as hardness and conductivity $[1,2,3,4,5,6,7,8,9,10,11,12]$. Through methods such as density functional theory (DFT), the electronic proprieties of 
graphene can be obtained with a considerable level of precision. However, the Ab initio model requires a considerable computational cost when dealing with considerably large systems, once the solution is obtained from the Schroedinger equations. Based on a Hamiltonian model, the tight-binding model (TB) has been used as an alternative way for the calculation of band structure with larger systems in a practical manner. However, until now, there is no free software specialized in solving a generalized eigenvalue problems based on hamiltonian model for complex carbon-based systems

Classical molecular dynamics (MD) is a low computational cost method compared to Ab initio models. Through molecular dynamics, it is possible to obtain thermodynamic properties of statistical ensembles under various temperature and pressure conditions. Moreover, the time evolution of canonical variables such as position and momentum of each particle can be determined with a certain degree of precision at low computational cost, compared to Ab initio models. Due to this characteristic, the MD allows for getting an analysis of the physical properties of condensed systems such as the assignment of vibrational modes, diffusion, free energy, etc. For this purpose, diverse programs have been developed in order to perfectly perform the MD model of condensed systems $[13,14,15]$.However, until now, there is no known software capable to get physical properties of carbon-based nanostructures by two different techniques (MD and TB).

In this work, a widely functional platform capable of simulating carbon-based nanostructures and other condensed systems using low-cost simulation techniques will be presented. Among them, we mention tight-binding and molecular dynamics. In order to test the reliability of the results obtained by the HICOLM program, the graphene band structure was calculated using the TB model, including a Hamiltonian model with the dependence of the relative distance between neighboring atoms and the hybridization effect of the $\mathrm{s}$ and $\mathrm{p}$ orbitals. To test the reliability of the program in the execution of the MD of systems composed of interacting molecules, simulations of condensed systems were carried out, such as the supercritical phase of argon and liquid water under various temperature and pressure conditions. The calculated values were compared with experimental data and other theoretical results found in the literature. The HICOLM program is available under the GPL free software license and can be downloaded through the GITHUB platform (https://github.com/flavianowilliams/HICOLM).

\section{Theory}

\subsection{Tight-binding}

The trouble of solving the generalized eigenvalue problem, which is the basis of the TB method is to get the values of $\lambda$ that satisfies the following matrical equation

$$
\mathbf{H} \cdot \mathbf{x}=\lambda \mathbf{S} \cdot \mathbf{x} .
$$

$\lambda$ depicts the eigenvalues which are the energy level of electrons at each k-point in the Brillouin zone. The element of matrix in Eq. 1 are defined as

$$
\begin{aligned}
h_{l_{i} \mu_{i}, l_{j} \mu_{j}} & =\sum_{m_{i}, m_{j}, m^{\prime}} c_{\mu_{i} m_{i}}^{l_{i} *} c_{\mu_{j} m_{j}}^{l_{j}} d_{m^{\prime} m_{i}}^{l_{i}^{*} *} d_{m^{\prime} m_{j}}^{l_{j}} V_{l_{i} l_{j} m^{\prime}}, \\
s_{l_{i} \mu_{i}, l_{j} \mu_{j}} & =\sum_{m_{i}, m_{j}, m^{\prime}} c_{\mu_{i} m_{i}}^{l_{i} *} c_{\mu_{j} m_{j}}^{l_{j}} d_{m^{\prime} m_{i}}^{l_{i}^{*} *} d_{m^{\prime} m_{j}}^{l_{j}} S_{l_{i} l_{j} m^{\prime}}
\end{aligned}
$$

where $\mu=1, \ldots, l+1$ e $l$ are the angular moment of each atomic orbital, $l=0,1$. $h$ and $s$ are evaluated by the sum of the magnetic quantum number $m$, where $m=[-l, l]$ of the atoms $i$ and $j$ and $m^{\prime}=\left[\min \left(l_{i}, l_{j}\right), \max \left(l_{i}, l_{j}\right)\right] . c_{\mu m}^{l}$ depicts the overlap $|l, m\rangle$ onto $|l \mu\rangle$,

$$
|l \mu\rangle=\sum_{m=-l}^{m=l} c_{\mu m}^{l}|l, m\rangle .
$$

$V_{l_{i} l_{j} m^{\prime}}$ e $S_{l_{i} l_{j} m^{\prime}}$ are called Slater-Koster parameters (SK), where $m^{\prime}$ depicts the coupling atomic orbitals hybridization $\sigma, \beta, \delta$. In a relative distance-dependent hamiltonian model $h_{l_{i}} \mu_{i}, l_{j} \mu_{j}$ and $s_{l_{i}} \mu_{i}, l_{j} \mu_{j}$ are empirically defined by Eqs. 5 and 6 ,

$$
\begin{aligned}
& V_{l l m}(r)=f(r)\left(\beta_{l l m}^{1}+\beta_{l l m}^{3} r\right) e^{-\beta_{l l m}^{2}}, \\
& S_{l l m}(r)=f(r)\left(\gamma_{l l m}^{1}+\gamma_{l l m}^{3} r\right) e^{-\gamma_{l l m}^{2}} .
\end{aligned}
$$

The dependence of the relative distance between two atoms in the hybridization is defined by the variable $r$ and the parameters which define the SK values for each orbital are $\beta_{l l m}$ and $\gamma_{l l m}$. The range of atomic orbitals is defined by Eq. 7 ,

$$
f(r)=\frac{1}{1+e^{-\frac{\left(r-r_{c}\right)}{\kappa}}} .
$$

$\kappa$ depicts the occupation of electronic states simulated at a certain temperature in a Fermi-Dirac distribution, and it defines the smoothness of function around the cutoff radius $r_{c}$.

The onsite terms are defined by Eq. 8,

$$
\varepsilon_{l, m}=f(r)\left(\alpha_{l, m}^{1}+\alpha_{l, m}^{2} \rho+\alpha_{l, m}^{3} \rho^{2}\right) .
$$

$\rho$ depicts the dependence of each orbital 1 with electronic density and $\alpha_{l, m}$ are parameters for the onsite values of each atomic orbital.

\subsection{Molecular dynamics \\ 2.2.1 MD cycle}

The main idea of the MD model involves concepts related to the dynamics of the participants that make up the physical system at a atomic level, where the canonical variables (position and momentum) of each atom can be determined by using the integrator of movement called Velocity Verlet $[16,17]$. These method raises the position $\mathbf{r}$ and velocity $\mathbf{v}$ coordinates of each atom from the time t to $t+\delta t$, according Eqs. 9 e 10 ,

$$
\begin{aligned}
\mathbf{r}(t+\delta t) & =\mathbf{r}(t)+\delta t \mathbf{v}(t)+\frac{1}{2 m} \delta t^{2} \mathbf{F}(t) \\
\mathbf{v}(t+\delta t) & =\mathbf{v}(t)+\frac{1}{2 m} \delta t[\mathbf{F}(t)+\mathbf{F}(t+\delta t)]
\end{aligned}
$$


However, the updating of the list intra e intermolecular interacting forces consumes about $40 \%$ of the time at each MD cicle, rising the computational cost [14]. To decrease this consume, strategies, such as Verlet neighbour list [16], has been used as an alternative way. Considering the Van der Waals is a short-ranged interaction, we can say with a good approximation that only atoms at a certain distance, let say $r_{c}+d r_{c}$, where $r_{c}$ is the cutoff radius, will be effectively interact with their near neighbour. For those who are beyond the cutoff radius, their contribution might be neglected. Thus, a list of nearby neighbors called Verlet neighbor list is built for each system atom. As long as the timestep is relatively small, we might consider that the Verlet list remains largely unchanged for a relatively long time. The updating of Verlet list is performed at each interval verlchk, where

$$
\text { verlchk }=\frac{d r_{c}}{\left|\mathbf{v} \delta t+\frac{\mathbf{F}}{2 m} \delta t\right|} .
$$

$m$ depicts the atomic mass, $v$ the velocity and $F$ is the force of each atom. $\delta t$ depicts the timestep, that must be indicated at the beginning of simulation.

\subsubsection{Force field}

The force field that defines the interaction between atoms that make up a condensed system is defined by a conjunction of intramolecular and intermolecular interactions, Eq. 12,

$$
\begin{aligned}
U & =\sum_{\substack{i=1 \\
j<i}} U_{i j}^{\text {coul }}+U_{i j}^{v w}+\sum_{\text {bond }} U^{\text {bond }}+\sum_{\text {bend }} U^{\text {bend }}+ \\
& +\sum_{\text {tors }} U^{\text {tors }}+\sum_{\text {Tersoff }} U^{\text {Tersoff }} .
\end{aligned}
$$

Long-range intermolecular interactions are represented by coulomb interaction between atoms due to their partial charges of the nucleus, and could be calculated in codensed systems through the damped cutoff-neutralized model proposed by Fenell et al. [18, 19, 20]. The Fennel model regards the effect of charge spread in a shield cloud depicted by a gaussian distribution and thus the coulomb interaction decreases at a larger distance. This reduces the effective interaction of potential up to the cutoff radius, $r_{c}$. Eq. 13 shows the Fennel potential [19],

$$
\begin{aligned}
U_{i j}^{\text {coul }}(r) & =q_{i} q_{j}\left[\frac{\operatorname{erfc}(\alpha r)}{r}-\frac{\operatorname{erfc}(\alpha r)}{r_{c}}+\right. \\
& +\left(\frac{\operatorname{erfc}\left(\alpha r_{c}\right)}{r_{c}^{2}}-\frac{2 \alpha}{\sqrt{\pi}} \frac{e^{\left(-\alpha^{2} r_{c}^{2}\right)}}{r_{c}}\right) \times \\
& \left.\times\left(r-r_{c}\right)\right]
\end{aligned}
$$

erfc is a complementary error function, which simulates the effect of shield around each particle.

To get the Van der Waals interaction, the Lennard-Jones, Eq. 14, and Morse, Eq. 15, might be employed,

$$
\begin{aligned}
U_{\mathrm{LJ}}^{\mathrm{VW}}(r) & =4 x_{1}\left[\left(\frac{x_{2}}{r}\right)^{12}-\left(\frac{x_{2}}{r}\right)^{6}\right], \\
U_{\text {Morse }}^{\mathrm{VW}}(r) & =x_{1}\left[e^{-2 x_{2}\left(r-x_{3}\right)}-2 e^{-x_{2}\left(r-x_{3}\right)}\right] .
\end{aligned}
$$

The Lennard-Jones function is a good choice for systems that contains weak interactions, such as gas phase or liquid systems, due to their simplicity and the small quantity of parameters has to be adjusted. Another option is the Morse potential, very useful for molecules to reproduce anharmonic chemical bonds.

The intramolecular interaction are depicted by bond, bending and dihedral interactions. To bond interaction, two options are possible: Morse, Eq. 16, and harmonic potential, Eq. 17,

$$
\begin{aligned}
U_{\text {Morse }}^{\text {bnd }}(r) & =y_{1}\left[e^{-2 y_{2}\left(r-y_{3}\right)}-2 e^{-y_{2}\left(r-y_{3}\right)}\right], \\
U_{\text {Harm }}^{\text {bnd }}(r) & =\frac{y_{1}}{2}\left(r-y_{2}\right)^{2} .
\end{aligned}
$$

The harmonic potential is also used to represent the bending and dihedral interactions.

To represent covalent interactions between carbon and hydrogen atoms, such as hydrocarbons and carbon-based nanostructures, the Tersoff model adapted by Brenner et all $[5,21]$ might be employed. Eq. 18 shows the Tersoff potential,

$$
U^{\text {Tersoff }}=\sum_{i} \sum_{j \neq i} f_{c}\left(r_{j i}\right)\left[V_{R}\left(r_{j i}\right)-\bar{b}_{i j} V_{A}\left(r_{j i}\right)\right]
$$

$V_{R}$ depicts the repulsion term of the potential and $V_{A}$ is the atraction term. $\bar{b}_{i j}$ measures the intensity of the atraction term, in which depends on the number of bonds shared for each atoms in the structure, where

$$
\bar{b}_{i j}=\frac{1}{2}\left(b_{i j}+b_{j i}\right) .
$$

$f_{c}$ is the cutoff function, which cuts off the action to only nearly neighbour atoms,

$$
f_{c}(r)= \begin{cases}1, & r \leq t_{1} \\ {\left[1+\frac{1}{2} \cos \left(\pi \frac{r-t_{1}}{R_{2}-t_{1}}\right)\right],} & t_{1}<r \leq t_{2} . \\ 0, & r>t_{2} .\end{cases}
$$

$V_{A}$ e $V_{R}$ represent the respective terms of attraction and repulsion between atomic pairs, which are performed by functions similar to the Morse potential, as demonstrated by Eqs. 21 and 22,

$$
\begin{aligned}
& V_{A}(r)=\frac{t_{3} t_{5}}{t_{5}-1} e^{-\sqrt{\frac{2}{t_{5}}} t_{4}\left(r-t_{6}\right)}, \\
& V_{R}(r)=\frac{t_{3}}{t_{5}-1} e^{-\sqrt{2 t_{5}} t_{4}\left(r-t_{6}\right)} .
\end{aligned}
$$

\subsubsection{Minimum image convention}

The MD model is usually employed in systems with a limited amount of atoms and molecules that undergo the surface effects caused by the interface between liquid and void. In order to represent real systems, where it is desired to provide information about system in their bulk state, a method called minimum image convention is very useful in MD calculus. In this one, a supercell can be considered as sufficiently as large so that we can project with good approximation only 
the neighboring atoms that lie at the edges of unit cell. Such a procedure is justifiable if we consider short-ranged interactions, such as intramolecular forces and Van der Waals among molecules. In an orthorhombic cell, where the atom in neighborhood of another one is projected to its next image, the relative distance between them is defined according the following relations

$$
\begin{aligned}
& \delta x=\delta x-a \cdot \operatorname{int}\left(\frac{2 \delta x}{a}\right), \\
& \delta y=\delta y-b \cdot \operatorname{int}\left(\frac{2 \delta y}{b}\right), \\
& \delta z=\delta z-c \cdot \operatorname{int}\left(\frac{2 \delta z}{c}\right),
\end{aligned}
$$

where $a, b$ e $c$ are lattice constants and $\delta r$ is the relative distance. However, it is important the value of lattice constants do not overcome the double of cutoff radius $r_{c}$, to avoid any undue counting of each atom with their own image.

\subsubsection{Statistical ensemble}

In the MD simulation of condensed systems at thermodynamic equilibrium, some statistical ensembles might be evaluated by controlling the temperature and pressure through the time of simulation. For instance, we can cite three statistical ensembles: NVE, NVT and NPT. These initials represents three variables which remains unchanged during the time of simulation. $\mathrm{N}$ represents the number of molecules, $\mathrm{V}$ is the volume of simulation box size, $\mathrm{T}$ is the temperature and $\mathrm{P}$ the pressure of system, which is evaluated during the time of simulation. For this, diverse algorithms of controlling could be used to control the temperature and pressure, where the most common is the Berendsen algorithm [22].

\section{Hicolm structure}

\subsection{Overal workflow}

Fig. 1 shows the overal workflow of the HICOLM program. basically, the program is split into two parts, the first one corresponds to the description of the physical system which will be simulated. This part, the user must furnish information about the structure, such as the vector coordinates of the unit cell, the number of atoms and information regarding to each atom (atomic number, spacial coordinates and type). The second one, corresponds to the model to be employed (TB or $\mathrm{MD})$, where the user must furnish information relevant to each one. After the choice of the model, HICOLM program might acquires several physical proprieties. In the TB model, three physical proprieties related to the band structure and electronic energy could be calculated by HICOLM program. In the MD model, canonical variables for each atom at each elapsed time are calculated in three statistical ensembles (NVE, NVT and NPT). These values are remained in a output file called HICOLM.md, where several physical proprieties, such as thermodynamic, energetic, vibrational and structure might be acquired by using the utilities that are included in the HICOLM package.

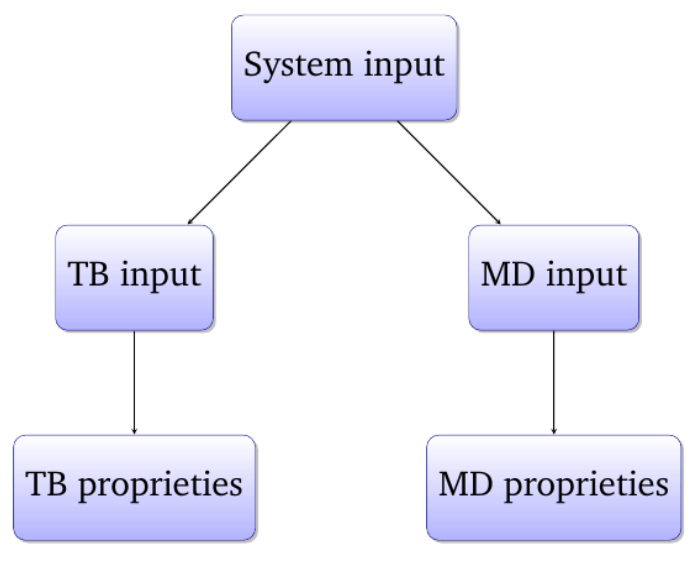

Figure 1. Overall workflow of HICOLM program.

\subsubsection{Units of measurement}

The HICOLM program works with two systems of units, the entrance units and the internal units. The entrance unit are associated with the variables provided by the user at the beginning of the simulation, through the HICOLM.in file. The units of the variables, printed in the HICOLM.out file, are the same as those used in the entrance units. The internal unit are based on the fundamental units of the atomic unit system (a.u.), and are used by the program throughout simulation for the development of calculations. Tab. 1 shows the entrance and internal units of the variables used in HICOLM program and their corresponding conversion factors.

\subsection{Input and their description}

Input 1 shows the directives referring to the definition of the system to be analyzed. These parameters are used in all models in HICOLM program (in this case, TB or MD) and must be included in the HICOLM.in input file. In Sec. \&INIT, the variable method provides the choice of method to be used. TB depicts the tight-binding method, and MD is the molecular dynamics method. Sec. \&CELL must contain the coordinates of the vectors of the unit cell. In Sec. \&COORD information regarding the system to be analyzed should be provided. The directive reuse is associated with the reading of structure values. Option 1 allows the program to read the predefined value of the unit cell, position, and strength of each atom furnished in the HICOLM.XSF file. The directive natom indicates the number of atoms in the unit cell. Next, the information of each atom must be provided. First, you must indicate its atomic number (variable $\mathrm{Z}$ ), followed by the atomic coordinates ( $\mathrm{x}, \mathrm{y}$ and $\mathrm{z}$ ). The last value corresponds to the variable $E_{a}$, which identifies the species to which the atom belongs.

Input 2 shows Sec. \&TBIND related to adjustment or pre defined set-up of the SK parameters necessary to achieve the $h_{l_{i} \mu_{i}, l_{j} \mu_{j}}$ and $s_{l_{i} \mu_{i}, l_{j} \mu_{j}}$ values. The parameters of Eqs. 5 and 6 can be automatically defined through the Powell hybrid optimization method, which is included in Minpack library 
HICOLM: High-Performance Platform of Large Systems by Using Low Computational Cost Methods

Table 1. Units of measurement used in the HICOLM program.

\begin{tabular}{lccl}
\hline Propriety & Entrance unity & Internal unity & Conversion \\
\hline \hline Length & $\AA$ & $a_{0}$ & $\left(\frac{\hbar^{2}}{m_{e} \hbar^{2}}\right)\left[10^{-10}\right]$ \\
Energy & Electron-volt & $E_{h}$ & $\left(\frac{m_{e} K^{2} e^{4}}{\hbar^{2}}\right)\left[6,241506 \times 10^{18}\right]$ \\
Pressure & Atmosphere & $\left(\frac{E_{h}}{a_{0}^{3}}\right)$ & $\left(\frac{E_{h}}{a_{0}^{3}}\right)\left[0.9872 \times 10^{-5}\right]$ \\
Mass & Atomic mass & $m_{e}$ & $\left(\frac{m_{e}}{N_{0}}\right)\left[10^{3}\right]$ \\
Time & Picoseconds & $\left(\frac{\hbar}{E_{h}}\right)$ & $\left(\frac{\hbar}{E_{h}}\right)\left[10^{12}\right]$ \\
Temperature & Kelvin & $\frac{E_{h}}{k_{B}}$ & $\frac{E_{h}}{k_{B}}$ \\
Charge & $\mathrm{e}$ & $e$ & 1 \\
Angle & Degree & $\mathrm{rad}$ & $\frac{180}{\pi}$ \\
\hline
\end{tabular}

Input 1 Parameters of structure of the system.
\&INIT
method (TB or MD)
\&END
\&CELL
$v_{1} x v_{1} y v_{1} z$
$v_{2} x v_{2} y v_{2} z$
$v_{3} x v_{3} y v_{3} z$
\&END
\&COORD
reuse $(0$ or 1$)$
natom $N_{a}$
$Z x y z E_{a}$
$\cdots$
\&END

$[23,24]$. Therefore, the user must choose the option $\mathrm{T}$ instead of $\mathrm{F}$ in the optimization variables found in HICOLM.in file. Thus, the only parameters with option $\mathrm{T}$ will be adjusted and the others, with option F, will be remained according the predefined values in the input file. diagop is the directive responsible for the diagonalization option, where the user has the freedom to choose between two diagonalization options of the secular matrix. Option 1 represents the choice in solving the generalized eigenvalue equation from the solution of a complex matrix, while option 2 represents the choice in solving the eigenvalue equation of the real matrix. Both cases use the subroutines provided by the lapack library [25]. Simulations with graphene demonstrated that the choice of option 2 resulted in higher computational cost, however, also revealed greater numerical stability in the diagonalization of the secular matrix compared to option 1. rcutt is the directive responsible for defining the shear radius of the Fermi-Dirac distribution, and lcutt a smoothness of Eqs. 2 and 3 near the $r_{c}$. The directive dens depicts the contribution of electronic density in the calculation of the $\varepsilon_{l, m}$ energy of each atomic orbital. The optimization of the parameter $\rho$ can be obtained through the variable $O_{\rho}$ (T ou F). Next, we have the directive nonst that defines the number of the atomic orbitals. In the next line, it necessarily follows the identification of each onsite term, given by the quantum numbers 1 and $\mathrm{m}$. The number of parameters to be used is given by the variable $N_{\varepsilon}$, referring to onsite terms, Eq. 8. The quantity of the offsite terms to be used in the simulation is defined by the directive nsk. The following lines indicate the sections referring to each SK value and their respective parameters. The optimization of those parameter can be defined by the variables $O_{\text {off }}\left(\mathrm{T}\right.$ ou F). $N_{S K}$ indicates the number of parameters to be used in Eqs. 2 and 3, followed by the lines that correspond to the values has to be used.

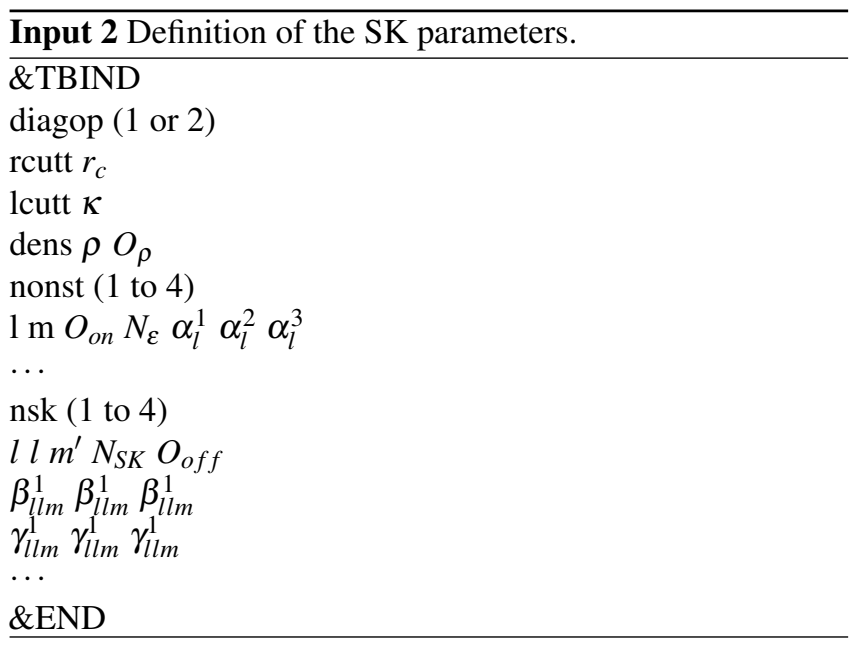

The dimension of the matrix is defined in section \&ORBIT, see Input 3. nlo indicates the orbitals that will be used in the TB simulation. Next, each value of the quantum number 1 must be put followed by their respective magnetic number $\mathrm{m}$, where the quantity is defined by the variable $N_{o}$. Input 3 also show the section OPTIM, responsible to carry the directives about the adjustment of the energy levels calculated in the TB method with values obtained in the DFT technique. nopt is the directive related to the adjustment of the parameters so that the energy values of each corresponding level coincide with 
the results obtained by the DFT technique (nopt $=1)$. If it is not convenient, the value 0 of nopt discards the optimization option, and the predetermined values in the input file will be used a priori. nkrlx indicates the number of bands that will be used for the adjustment. The first and second values indicate the order of bands of the calculation TB and DFT respectively, followed by their respective k-points. The number of k-points at each selected band should be presented in the third column (it is important to note that the total number of k-points must be equal to the number of SK parameters to be optimized).

Input 3 Definition of the orbitals that will be used in the TB
simulation and the k-points choosed to the adjustment of the
energy levels related to the DFT technique.
\&ORBIT
nlo $(1$ to 2$)$
$1 N_{o}$ m
$\&$ END
\&OPTIM
nopt $(0$ or 1$)$
nkrlx $(1$ to 10$)$
$n_{D F T} n_{T B} N_{f i t} k_{f i t}$
$\ldots$
$\& E N D$

Input 4 shows Sec. PROPER, which has the options related to the proprieties that must be furnished by the TB simulation. prop indicates the proprieties that will be calculated. The option EBANDS determines the calculus of the electronic energy and the option BANDS determines the calculus of band structure along the high-symmetry in the Brillouin zone. If the choice is the option BANDS, a highsymmetry line must be defined by the user, after the directive nband, that indicates the number of vectors in the Brillouin zone. bandopt depicts the unity of measurement to be used in the simulation. The option 1 represents the k-points in unities of $\pi / a$, and the option 2 represents the k-points in unities of measurement in vectors $\mathrm{K}$ of the reciprocal lattice. The option BANDS3D determines the calculus of the energy levels $E(k)$ in the Brillouin zone, where $\vec{k}=\left(k_{x}, k_{y}, k_{z}\right)$. The directive mesh determines the number of k-points for the grid to be considered at each axis in the Brillouin zone and the directive kptt0 determines the shift in the k-points throughout the Brillouin zone.

Input 5 shows the directives related to the MD method. The directive nhist indicates the quantity of frames to be recorded in the HICOLM.AXSF output file. This option is useful for the visualization and the time evolution of the physical system, and a corresponding animation can be made by the user. The directive preext relates the external pressure defined in unity of atmosphere that the system can undergoes and the directive text relates the temperature defined in Kelvin. ntrialmax relates the total number of MD steps along the simulation, whereas the directive nrelax defines the number of

Input 4 Definition of the parameters of the proprieties of the
TB method.
\&PROPER
prop (EBAND, BANDS or BANDS3D)
bandopt ( 1 or 2$)$
nband (1-10)
$N_{b} k_{1} k_{2} k_{3}$
$\ldots$
mesh (up to 250$)$
kptt0 $k_{0} x k_{0} y k_{0} z$
$\&$ END

steps carry out the relaxing process. The directive ensemble enables the choice of ensemble to be used in the simulation. If the option would be NVT, the directive $\tau_{T}$, associated to the temperature control through the thermostat Berendsen must be furnished. If NPT would be, the directive $\tau_{p}$, associated to the control of pressure through the barostat Berendsen must be furnished, joined to the $\beta$ factor, related to the isothermal compressibility of the system. For liquids, it os common to consider $\beta=4,9 \times 10^{-4} \mathrm{~atm}^{-1}$. The directive rcutoff is associated with the cutoff radius used for the long range correction of the energy and virial pressure, due to the shortrange effect of the Van der Waals interaction. Next, the value of the cutoff radius $r_{c}$ and the width of the Verlet neighbour list, $d r_{c}$, must be furnished by the user. Small values of $d r_{c}$ implies a larger updating of the Verlet neighbour list, which in turn implies an increase of the computational cost and the time of simulation. Moreover, for simulations, such as gas phase systems, small value of $d r_{c}$ might be necessary.

\begin{tabular}{l} 
Input 5 Parameters used in the MD method. \\
\hline MD \\
nhist $N_{s t p}$ \\
preext $p$ \\
text $T$ \\
ntrialmax $N_{T}$ \\
nrelax $N_{R}$ \\
timestep $d t$ \\
ensemble (nve, nvt or npt) $\tau_{T} \tau_{p} \beta$ \\
rcutoff $r_{c} d r_{c}$ \\
\&END
\end{tabular}

In Sec. FORCE, Input 6, the parameters of the Van der Waals and coulomb interactions are presented. The directive elect enables to define the partial charge $q_{a}$ of each atomic specie $E_{a} . N_{\text {elec }}$ indicates the number of atomic species that it have not null partial charge. vdw is the directive related to the Van der Waals interaction, where $N_{v w}$ indicates the number of paired interactions in the force field. Following, the user must furnish the lines related to each interaction. The two first values are the pair of species which will be defined by the user according to the Van der Waals potential, and $O_{v w}$ depicts the kind of potencial to represent the Van der Waals potential. Next, the parameters of Van der Waals potential 
must be furnished, according to Eqs. 14 to 15. The Tersoff potential might be included through the directive tersoff. $N_{\text {trsf } f}$ indicates the number of Tersoff potentials to be used in the force field (it is common to consider $N_{\text {trsff }}=1$ in simulations containing only carbon nanoestrutures). After that, the six parameters of the Tersoff potential must be provided.

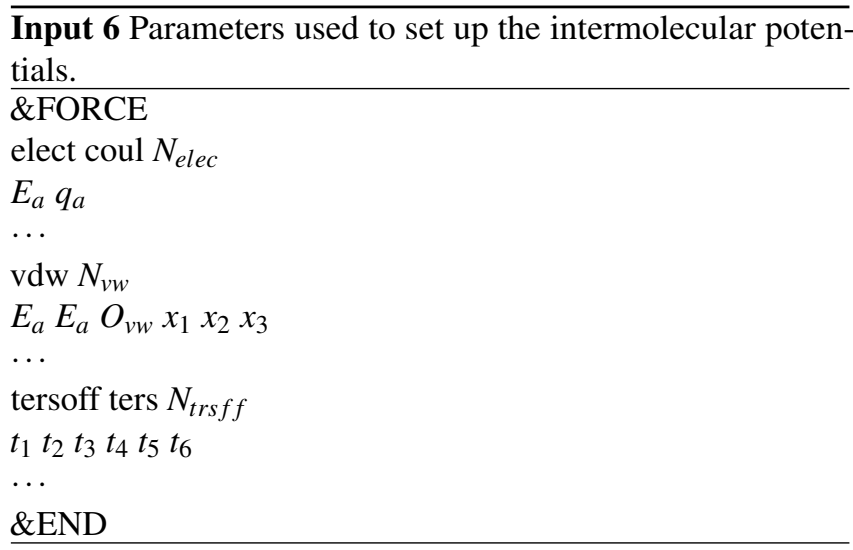

In Sec. MOLEC, Input 7, the parameters related to the geometry of molecules are presented, followed by those parameters of the intramolecular interactions. The directive molecs depicts the number os species that make up the condensed system, in accordance with the variable $N_{\text {molecs. }}$. The section that describes each molecule type must start with the variables $M_{i}, N_{i}$ and $Q_{i} . M_{i}$ indicates the name of the molecule, $N_{i}$ is the number of molecules of the corresponding specie and $Q_{i}$ is the number of atoms at each king of the molecule. Next, the user must furnish the parameters of the intramolecular interactions. The directive bends opens the section related to the corresponding parameters of the bending potential. $N_{b e n d s}$ depicts the number of bending interactions in the molecule. Next, the parameters of the bending potential must be presented. The Three next values depict the atoms in the bending interaction, where the atom $A_{i}$ is the central atom of the bending angle $A_{j}-A_{i}-A_{k}$. $O_{b e n d s}$ depicts the kind of potential to be used in the simulation. Nowadays, the HICOLM program furnishes only the harmonic potential to describe the bending interaction, Eq. 17. The next values depicts the parameters of bending potential. The directive bonds opens the section related to the parameters of bond potential. $N_{b n d s}$ represents the number of bond interaction in the molecule. After that, the parameters of each interaction must be furnished. The two next values depict atoms that make up the bond $A_{i}-A_{j}$ and $O_{b n d s}$ shows the kind of potential to be used in the simulation, Eqs. 16 or 17. The next values depict the parameters of bond potential. Finally, the directive dihedral opens the section related to the parameters of dihedral interaction. $N_{\text {tors }}$ depicts the number of potential to be used in the molecule. Next, the parameters of each interaction must be presented. The next four values depict atoms that make up the dihedral of each molecule, where those atoms $A_{j}$ e $A_{k}$ that make up the main axis of each dihedral must be in the second and fourth order among the four atomic identification. $O_{\text {tors }}$ depicts the kind of dihedral to be used in the simulation, however, to date, HICOLM program only furnish the harmonic potential. The author has been working to improve the force field by the inclusion of most potential kinds in the HICOLM program. The following values depict the parameters of dihedral potential.

Input 7 Parameters of each kind of molecule and their parameters of intramolecular potentials.

$$
\begin{aligned}
& \hline \& \text { MOLEC } \\
& \text { molecs } N_{\text {molecs }} \\
& M_{1} N_{1} Q_{1} \\
& \text { bends } N_{\text {bends }} \\
& A_{j} A_{i} A_{k} O_{\text {bends }} y_{1} y_{2} y_{3} \\
& \ldots \\
& \text { bonds } N_{\text {bnds }} \\
& A_{i} A_{j} O_{\text {bnds }} y_{1} y_{2} y_{3} \\
& \ldots \\
& \text { dihedral } N_{\text {tors }} \\
& A_{i} A_{j} A_{k} A_{n} O_{\text {tors }} y_{1} y_{2} y_{3} \\
& \ldots \\
& M_{2} N_{2} Q_{2} \\
& \text { bends } N_{\text {bends }} \\
& A_{j} A_{i} A_{k} O_{\text {bends }} y_{1} y_{2} y_{3} \\
& \ldots \\
& \text { bonds } N_{b n d s} \\
& A_{i} A_{j} O_{b n d s} y_{1} y_{2} y_{3} \\
& \ldots \\
& \operatorname{dihedral} N_{\text {tors }} \\
& A_{i} A_{j} A_{k} A_{n} O_{\text {tors }} y_{1} y_{2} y_{3} \\
& \ldots \\
& M_{n} N_{n} Q_{n} \\
& \ldots \\
& \& \text { END } \\
&
\end{aligned}
$$

\section{Computational procedure}

\subsection{Tight-binding \\ 4.1.1 Graphene}

The band structure of graphene was calculated using the option BANDS in HICOLM.in file and the following vectors was used to represent the unit cell,

$$
\vec{a}_{1}=\frac{a}{2}(3, \sqrt{3}), \quad \vec{a}_{2}=\frac{a}{2}(3,-\sqrt{3}),
$$

where $a=1,42 \AA$ the carbon-carbon bond length. In these case, the reciprocal lattice vectors are

$$
\vec{b}_{1}=\frac{2 \pi}{3 a}(1, \sqrt{3}), \quad \vec{b}_{2}=\frac{2 \pi}{3 a}(1,-\sqrt{3}) .
$$

From the reciprocal lattice vectors the k-points of the Dirac cones $K$ and $K^{\prime}$ could be determined, where

$$
\vec{K}=\frac{2 \pi}{3 a}\left(1, \frac{\sqrt{3}}{3}\right), \quad \vec{K}^{\prime}=\frac{2 \pi}{3 a}\left(1,-\frac{\sqrt{3}}{3}\right) .
$$


The SK parameters of graphene, Eqs. 5 to 8, was obtained through the adjustment of band structure calculated by TB model and their agreement the results of density functional theory (DFT), which was made using a optimization method performed by the Minpack library. The subroutine dgeev in the Lapack library was used to get a diagonalization of a real matrix of Eq. 1. To achieve the conventional model (nondistance dependent), twelve k-points in the Brillouin zone was choice and for the range of atomic orbitals, the values $r_{c}=1,5 \AA$ and $\kappa=0,01 \AA^{-1}$ was defined in Eq. 7. To achieve the onsite, offsite and overlaps terms, only the first terms of Eqs. 5, 6 and 8 was used. In the case of distance-dependent model, we choice twenty eight k-points in the Brillouin zone of valence and the first conduction bands. To the range of atomic orbitals, the values $r_{c}=2,1 \AA$ e $\kappa=0,25 \AA^{-1}$ was defined in Eq. 7. The value $\rho=940 e / \AA^{-3}$ was used to represent the electronic density $\rho$, which is used in Eq. 8 . The SK parameters found for distance-dependent TB model is showed in Tab. 2.

Siesta package [26] was used for the DFT calculus with pseudopotencial and localized LCAO basis-set wavefunctions. For that, the double- $\xi$ with polarization by the inclusion of the $d$ orbital in valence shell (DZP) in the carbon atoms was chosen to get an improved basis-set wavefunction. The method proposed by Dion et al was made to get the exchangecorrelation effects of pseudopotential. A maximum error of $10^{-5} e / \AA$ in density matrix was adopted for a convergence of wavefunctions. A modified Broyden algorithm was adopted to optimize both atomic coordinates and the unit cell and a maximum value of $0,01 \mathrm{eV} / \AA$ was adopted for the atomic forces.

\subsection{Molecular dynamics}

To check the precision of HICOLM program in the obtaining physical proprieties of condensed system, two distinct systems was choose and their results compared with theoretical and experimental data found in the literature.

\subsubsection{Argon}

To check the precision of HICOLM program in the analysis of the effects of Van der Waals interaction in condensed systems, the argon gas in supercritical conditions was made. The fluid argon gas is a good example of condensed system due to the simplicity of its force field, where we only used the LennardJones potential with values $\varepsilon=125.7 \mathrm{Ke} \sigma=3.345 \AA$ [27] to represent the intermolecular interactions. The system was composed by 250 argon molecules in an orthorhombic simulation box. The calculus was obtained during $100 \mathrm{ps}$ and $1 \mathrm{fs}$ as a timestep in the NPT ensemble after a previous relaxation of the system. The temperature and pressure was controlled at each $0.1 \mathrm{ps}$ by the thermostat and barostat, and to adjust the box size we used the value $4.9 \times 10^{-5} \AA^{-1}$ as isothermal compressibility in the Berendsen algorithm.

\subsubsection{Water}

To check the precision of HICOLM program in the analysis of physical proprieties of condensed system composed by a force field which includes intramolecular potentials, water was choice in its liquid phase. The spc-fw model proposed by $\mathrm{Wu}$ et al [28] was used to represent the force field of liquid water. The system was composed by 100 water molecules in an orthorhombic simulation box. The calculus was obtained during $100 \mathrm{ps}$ and $1 \mathrm{fs}$ as a timestep after a previous relaxation of the system. The results was obtained in the ensembles NPT and NVT, where the temperature and pressure was controlled at each 0.1 ps by the thermostat and barostat Berendsen. In the ensemble NVT, an orthorhombic simulation box was used with the size of $12.81 \AA$.

\section{Results and discussion}

\subsection{Graphene}

Fig. 2 shows the energy of band structure of graphene by the DFT (red solid line) calculus and the energy levels of the TB (dotted line) for graphene using the conventional model [29]. In this case, the only $p_{z}$ orbital was used to define the band structure of graphene. The red lines show the band structure of DFT results, which was chosen for the adjustment of the parameters of TB model. The energy levels of the electrons at the orbitals $\mathrm{s}$ and $\mathrm{p}$ was evaluated by the solving of Eq. 1 . Fig. 2 reveals the results found by HICOLM program was similar for those found by Konschuh et al [4], where the band structure was in a good agreement with the DFT calculus, except the valence $\sigma$ band and the higher conduction bands, especially at about the M k-point of second lower and the first conduction bands and the K-point of third valence band. Konschuh reveals the disagreement of the $\sigma$ band with DFT result is due to the absence of hybridization between the $\mathrm{s}$ and p orbitals, which is not included in the conventional model. According the $\pi$ band, Konschuh relates the disagreement at the M k-point with absence of interaction between each atom and its next-nearest neighbors. Except the conduction bands, the TB model agrees satisfactorily the valence bands with DFT results, except the values near the M point (third valence band and first conduction band, agreeing with Konschuh analysis) and the $\Gamma \mathrm{k}$-point (first an third valence and conduction bands). However, the band structure based on the conventional TB model showed a better result of the third valence band at the $\mathrm{K}$ point compared to the method proposed by Konschuh. This result agrees with the analysis of Konschuh, that the disagreement of TB model with DFT result at the $\mathrm{M}$ e $\mathrm{K} \mathrm{k}$-points is due to the lack of hybridizations between $s$ and $p$ orbitals. Therefore, HICOLM program, with a better description of eigenvalue equation, Eq. 1, with the inclusion of $\mathrm{s}$ and $\mathrm{p}$ orbitals is able to get a better results due to hybridization effects.

Fig. 3 shows the energy of the band structure of graphene by DFT results and the TB relative-distance dependent model. The red lines indicate the energy levels found DFT calculus and the dotted lines the TB model. Compared to conventional 
HICOLM: High-Performance Platform of Large Systems by Using Low Computational Cost Methods

Table 2. SK parameters of graphene using distance-dependent TB model in HICOLM program.

\begin{tabular}{cccccccccc}
\hline & \multicolumn{3}{c}{ onsite } & \multicolumn{3}{c}{ offsite } & \multicolumn{3}{c}{ overlap } \\
\cline { 2 - 9 } & $\alpha_{1}(\mathrm{eV})$ & $\alpha_{2}$ & $\alpha_{3}\left(\AA^{-1}\right)$ & $\beta_{1}(\mathrm{eV})$ & $\beta_{2}$ & $\beta_{3}\left(e V \AA^{-1}\right)$ & $\gamma_{1}$ & $\gamma_{2}$ & $\gamma_{3}\left(\AA^{-1}\right)$ \\
\hline$\varepsilon_{s}$ & $-13,5024$ & $+0,0000$ & $+0,0000$ & & & & & & \\
$\varepsilon_{p,-1}$ & $-6,5038$ & $+0,0000$ & $+0,0000$ & & & & & & \\
$\varepsilon_{p, 0}$ & $-4,3060$ & $+0,0000$ & $+0,0000$ & & & & & & \\
$\varepsilon_{p,+1}$ & $-5,1555$ & $+0,0000$ & $+0,0000$ & & & & & & \\
$V_{s s \sigma}$ & & & & $-4,2066$ & $+0,2634$ & $-1,3144$ & & & \\
$V_{s p \sigma}$ & & & & $+3,1716$ & $-0,0019$ & $+11,6482$ & & & \\
$V_{p p \sigma}$ & & & & $+4,5979$ & $+0,0000$ & $+0,0000$ & & & \\
$V_{p p \pi}$ & & & $-2,8091$ & $+0,0082$ & $-46,9758$ & & & \\
$S_{s s \sigma}$ & & & & & & $+0,0002$ & $+0,0012$ & $+0,0234$ \\
$S_{s p \sigma}$ & & & & & & $-0,0020$ & $+7,2697$ & $-0,0656$ \\
$S_{p p \sigma}$ & & & & & & $+0,0002$ & $+0,0000$ & $+0,0000$ \\
$S_{p p \pi}$ & & & & & & $+0,0183$ & +24.3959 & $+0,0406$ \\
\hline
\end{tabular}

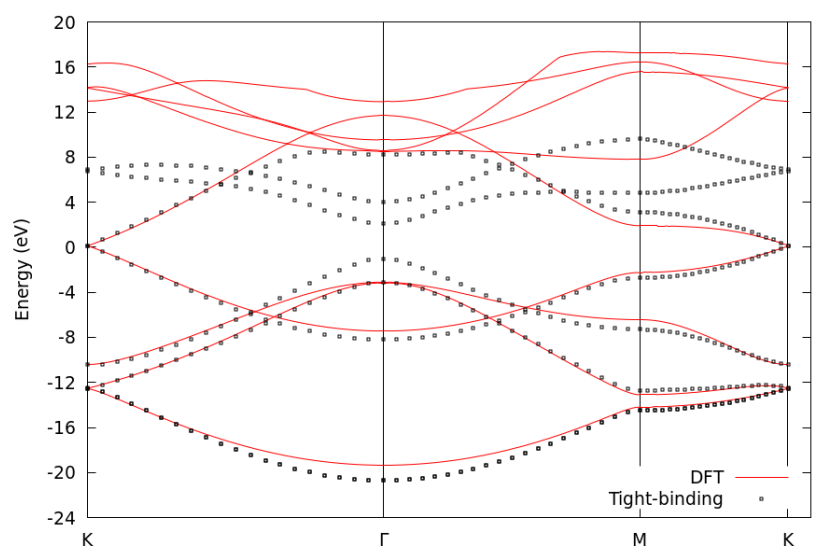

Figure 2. Band structure of graphene by DFT results (red solid line) and the conventional TB model (dotted line).

model, the relative-distance dependent model showed better results, mainly at the $\Gamma$ and $\mathrm{M}$ k-points in the $\sigma$ and $\pi$ bands. We believe this improvement is due to the inclusion of interactions between carbon atoms and their long-ranged neighbors (as suggested by Konschuh). Therefore, HICOLM program was able to get the proper correction by using Eqs. 5 to 8 .

Uma visão em três dimensões de cada nível de energia pode ser visualizada a partir da ferramenta gnuplot. A Fig. 4 mostra uma visão em perspectiva da banda de valência em perfil enquanto que a Fig. 5 mostra os mapas de contorno das quatro bandas de valência do grafeno. Os eixos x e y representam as coordenadas de cada k-point na zona de Brillouin, o eixo $\mathrm{z}$ representa a energia do estado eletrônico avaliada em cada k-point. Na Fig. 5, o mapa de cores mostra os valores de energia eletrônica avaliada em elétron-volt na zona de Brillouin. Em ambos os casos, é possível visualizar os cones de Dirac em torno de $K=(1,47 ; 0,85)$ e $K^{\prime}=(1,47 ;-0,85)$, estando de acordo com os valores da Eq. 28. Esse procedimento é útil caso seja desejável obter informações da localização dos cones de Dirac dentro da zona de Brillouin.

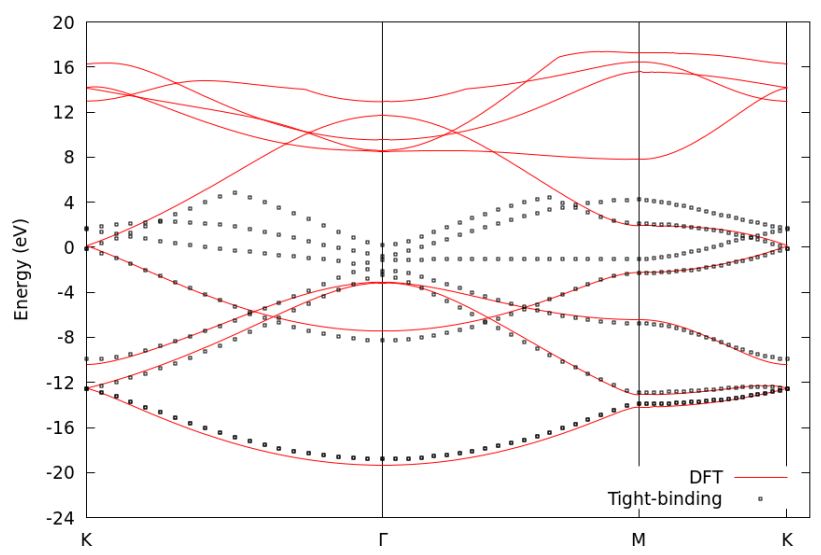

Figure 3. Band structure of graphene by DFT results (red solid line) and the relative-distance dependent TB model (dotted line).

A three dimensional view of each energy level can be viewed by using tools furnished in the gnuplot package. Fig. 4 shows a profile of the fourth valence band while the Fig. 5 shows the contour map of the four valence bands of graphene. The $\mathrm{x}$ and $\mathrm{y}$-axis are the k-point coordinates in the Brillouin zone and z-axis is the electronic energy evaluated at each kpoint. In Fig 5 the colour map shows the values of electronic energy evaluated in the Brillouin zone in the unit of electronvolts. In Both cases, it is possible to visualize the Dirac cones around the k-points $K=(1,47 ; 0,85)$ and $K^{\prime}=(1,47 ;-0,85)$, in accordance with Eq. 28 . This procedure is very useful to get information about the location of Dirac cones in the Brillouin zone.

\subsection{Argon}

Fig. 6 shows the CPU elapsed time for several values of the cutoff radius $r_{c}$. We can see an exponential increasing related to $r_{c}$, due to the quantity of neighbor atoms in Verlet neighbors list, which increases with the cutoff radius. Thus, this trend 


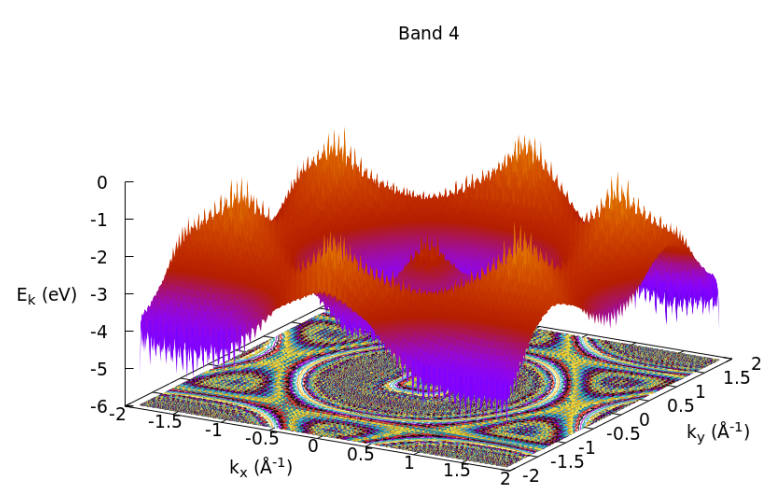

Figure 4. Profile of the fourth valence band of graphene. The $\mathrm{x}$ and $\mathrm{y}$-axis represents the $\mathrm{k}$-point coordinates in the Brillouin zone. $\mathrm{z}$-axis is the electronic energy evaluated at each k-point.
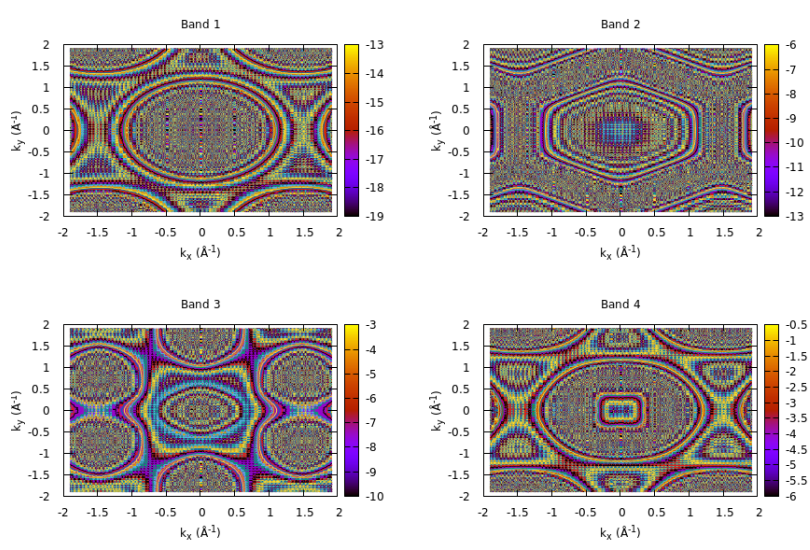

Figure 5. Contour maps of four energy levels in the valence bands of graphene. The colour map shows the values of electronic energy evaluated in the Brillouin zone in the unit of electron-volts.

also increase the quantity of interactions between pairs of atoms in neighboring molecules. Furthermore, we can also see the corrected term of Van der Waals interaction decreases, which converges to zero when $r_{c} \geq 10 \AA$.

Fig. 7 shows the fluctuation of total energy and the maximum variation of temperature $\Delta T$ found for several values of time constant $\tau_{T}$. In $\tau_{T} \leq 0,5 \mathrm{ps}$, the fluctuation of energy decreases followed by a meaningful rising for $\tau_{T}>0,5 \mathrm{ps}$. Furthermore, the inset graph shows values of $\Delta T$ related to the average temperature $(373 \mathrm{~K})$, where we can see those values increases with $\tau_{T}$. Therefore, caution must be taken with the choice of $\tau_{T}$, because small values lead to a greater control of temperature, however low values can also lead to an increase in the fluctuation of energy, as mentioned by Berendsen [22].

Fig. 8 shows the radial distribution function (RDF) of fluid argon at the conditions of $84,4 \mathrm{~K}$ and $1000 \mathrm{~atm}$. The dotted line shows the results of X-ray diffraction [30] while the solid line shows the results obtained by HICOLM program.

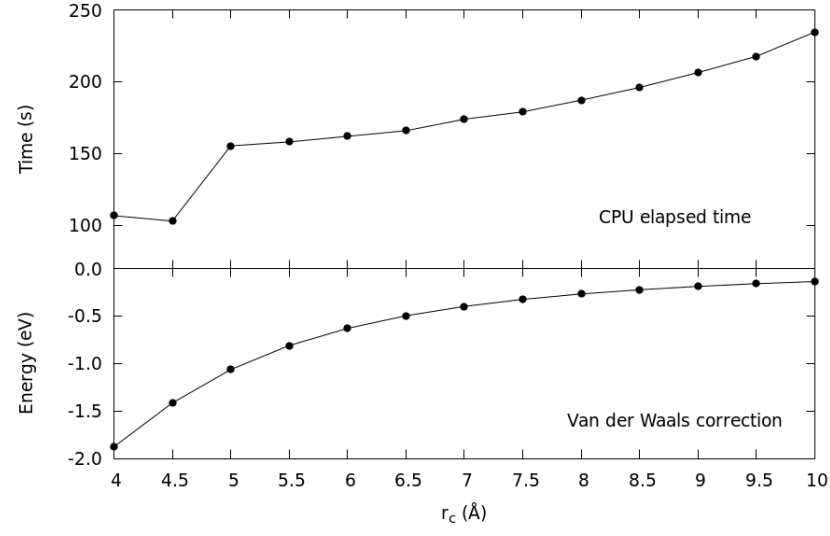

Figure 6. CPU elapsed time (top) and correction of Van der Waals energy as function of cutoff radius (bottom).

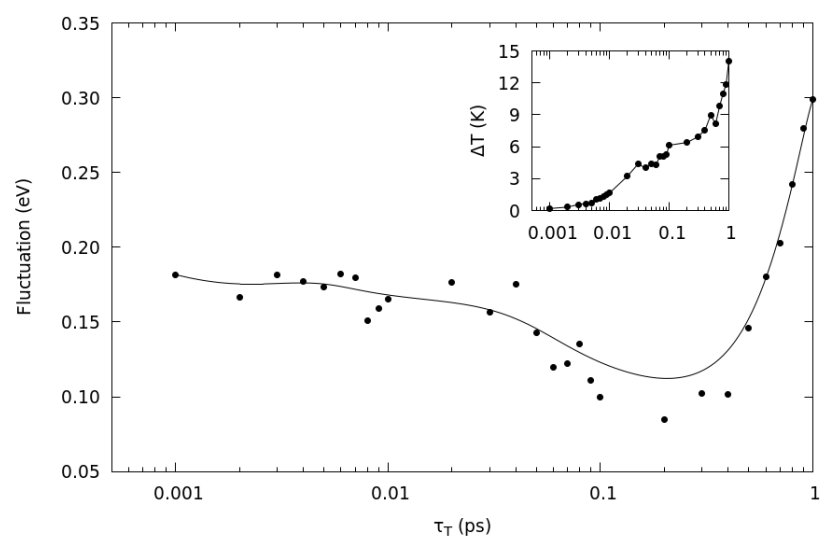

Figure 7. Average temperature for several values of time constant $\tau_{T}$.

RDF showed that the first coordination shell was found at around $3,6 \AA$. The density found for argon in fluid phase was $1,48 \mathrm{~g} / \mathrm{cm}^{3}$, where its experimental value corresponds to $1,43 \mathrm{~g} / \mathrm{cm}^{3}$ [31]. Therefore, the comparison of structure of liquid argon reveals a good agreement with other works found in the literature $[30,27]$. Therefore, it shows the HICOLM program is very capable to realize simulations of structures in their condensed phase through the Berendsen algorithm to control the pressure and temperature. RDF calculus can be performed by the utility proprieties furnished in HICOLM package.

Fig. 9 shows the average value of density for several pressure conditions at three isothermal curves $(273,298,373 \mathrm{~K})$ of the fluid argon gas. The black-dot lines represent the experimental values of the argon gas in its superfluid conditions and the red-dot lines represent the values found by HICOLM program. The fluctuation of density was practically null for higher pressure values compared to its mean value and a more meaningful ones for lower pressures, up to $200 \mathrm{~atm}$. The density was calculated for simulations with the cutoff radius $r_{c}=8 \AA$ and $r_{c}=10 \AA$. To lowest $r_{c}$ and higher pressures, we found the curve of density diverges from its experimental data 


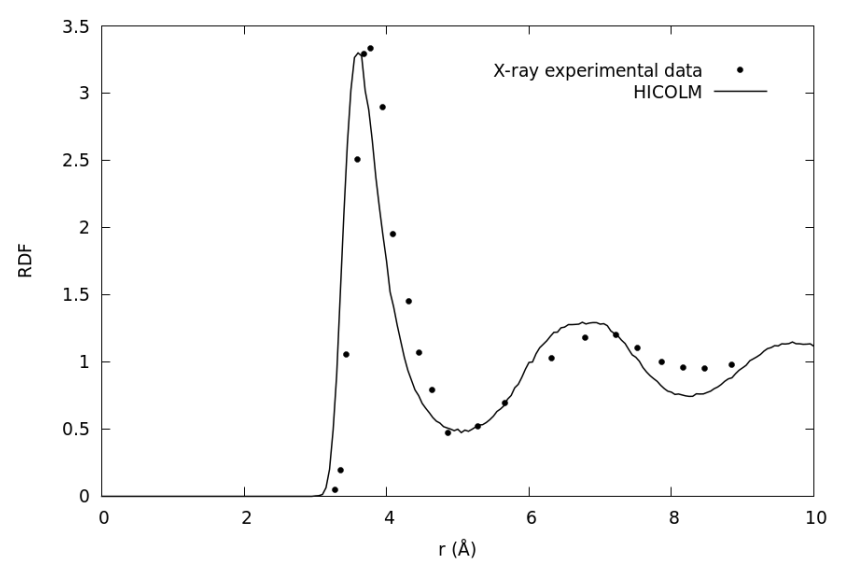

Figure 8. Radial distribution function of fluid argon at $84,4 \mathrm{~K}$ and $1000 \mathrm{~atm}$. X-ray experimental data (dotted line) and the results obtained by HICOLM program (solid line).

[32], and it converges next to 100 atm. Furthermore, estimated error of the result compared to experimental data rises meaningfully in cases where the cutoff radius is considerably small. However, this trend decreases to higher values of $r_{c}$, making the convergence of density going into the experimental data, even for higher pressures. To $r_{c}=10 \AA$, the estimated error was lower than two percents of the experimental data, rising to forty one percents at $100 \mathrm{~atm}$ and $273 \mathrm{~K}$ when $r_{c}=8 \AA$. In this case, it was not possible to observe any change in the density related to the temperature. Therefore, this analysis depicts the quality of simulation and its improvement with the cutoff radius. This fact is due to the effect of long-range correction of the coulomb interaction in the model proposed by Fennel, Eq. 13. Soon, we intent to implement more sophisticated methods, such as Ewald sum $[16,20]$ to improve the quality of the force field in HICOLM program.

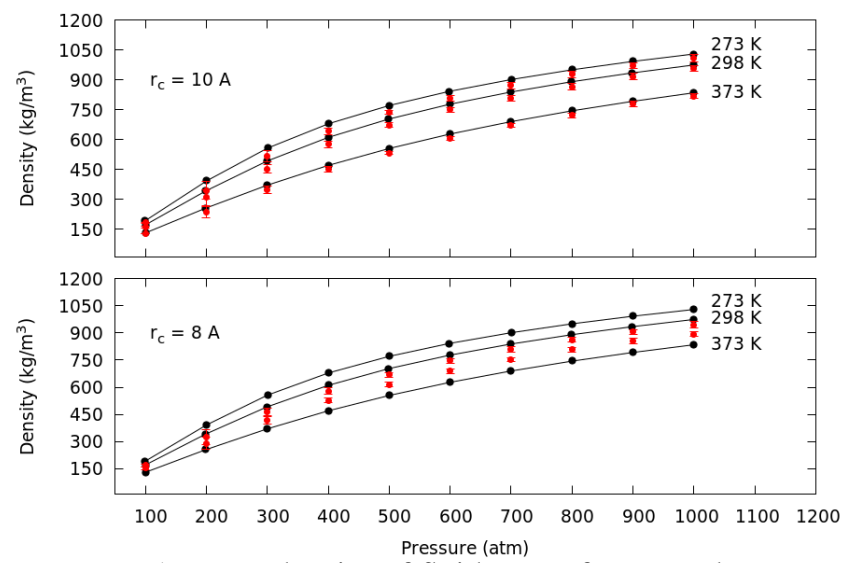

Figure 9. Average density of fluid argon for several pressure conditions at three isothermal curves.

\subsection{Water}

Fig. 10 shows the total energy found through the time simulation in the ensembles NVT e NPT at the pressure of $1 \mathrm{~atm}$ and several temperatures $(73,173,273$ and $373 \mathrm{~K})$. In all ensembles, we can see the lowest energy corresponds to the 73 $\mathrm{K}$, rising gradually with the increasing of temperature, which agrees the thermodynamic condition in statistical ensembles. Except for $373 \mathrm{~K}$, all NPT simulations yielded a lower total energy than their respective simulation in NVT ensemble. This trend was expected due to the fact that in ensemble NPT, the system is able to reproduce the effect of condensation of water and their properly relaxed volume at lower temperatures. This fact is not possible in ensembles NVT and NVE. Furthermore, all the simulation, the fluctuation in energy was lower than forty percent of its main value, showing that HICOLM program was able to reach the thermodynamic equilibrium after five picoseconds from the start of simulation.

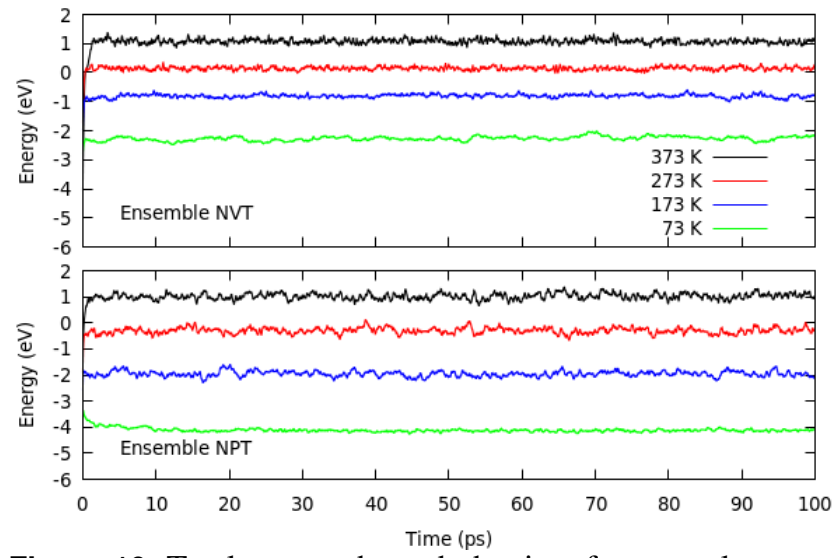

Figure 10. Total energy through the time for several temperatures in ensembles NVT and NPT.

HICOLM program also permits a vibrational analysis of atoms and molecules through the utility FTIR. For instance, normal modes such as stretch and scissors bending modes is also available in FTIR package, which turns HICOLM program a powerfull tool to analyze spectrum graphs and the assignment of vibrational modes. Autocorrelation function of atomic bonds is allowed by Eq. 30, which in turn it is associated with several stretch modes, while Eq. 31 permits for getting the angular autocorrelation function, which is associated with scissors vibrational mode of molecules. Eqs 29 depicts the average of velocity autocorrelation function of each atom $\mathrm{j}$ of specie $\mathrm{i}$ that make up the system. Eqs. 30 and 31 depicts the length and valence angle autocorrelation function of each molecule, respectively. Using the Fourier transform, HICOLM determines the respective vibrational mode, like stretch and scissors bending modes that might 
exist in a whole system.

$$
\begin{aligned}
& C_{a}(t)=\frac{1}{N_{i}} \sum_{j=1}^{N_{i}} \frac{\left\langle\vec{v}_{j}^{i}(t) \cdot \vec{v}_{j}^{i}(0)\right\rangle}{\left\langle\vec{v}_{j}^{i}(0) \cdot \vec{v}_{j}^{i}(0)\right\rangle}, \\
& C_{r}(t)=\frac{1}{N_{r}} \sum_{j=1}^{N_{r}} \frac{\left\langle r_{j}(t) \cdot r_{j}(0)\right\rangle}{\left\langle r_{j}(0) \cdot r_{j}(0)\right\rangle}, \\
& C_{\theta}(t)=\frac{1}{N_{\theta}} \sum_{j=1}^{N_{\theta}} \frac{\left\langle\theta_{j}(t) \cdot \theta_{j}(0)\right\rangle}{\left\langle\theta_{j}(0) \cdot \theta_{j}(0)\right\rangle} .
\end{aligned}
$$

The vibrational modes of the water molecule is well known in the literature, where the main bands are $3657 \mathrm{~cm}^{-1} \mathrm{e}$ $3756 \mathrm{~cm}^{-1}$ which are O-H symetric and asymetric stretch modes and $1595 \mathrm{~cm}^{-1}$ is the H-O-H scissors bending mode. Fig. 11 shows the atomic vibrational analysis of the oxygen atom (green line) e hydrogen (blue line). Fig. 11 also shows the scissors (red line) and stretch modes (black line). The inset graph shows the autocorrelation functions of each vibrational mode. It is possible to assign the peak at $1455 \mathrm{~cm}^{-1}$ for scissors vibration, once the oxygen, hydrogen and scissors curves match each other in this wavenumber. Next, the peak at $1525 \mathrm{~cm}^{-1}$ was found and their value correspond the combination of two bands, scissors and rotational modes (libration). Moreover, we can also identify two peaks at higher wavenumbers $\left(3610 \mathrm{~cm}^{-1}\right.$ e $\left.3733 \mathrm{~cm}^{-1}\right)$. The first one is the asymetric stretch while the second is the symetric stretch mode. As in scissors, we can assign this band to $\mathrm{O}-\mathrm{H}$ stretch modes because the matching among the oxygen, hydrogen and stretch curves. Therefore, we can say that the procedure adopted by HICOLM program to the assignment of normal modes permits to identify all the vibrational bands that might occurs in a complex molecule.

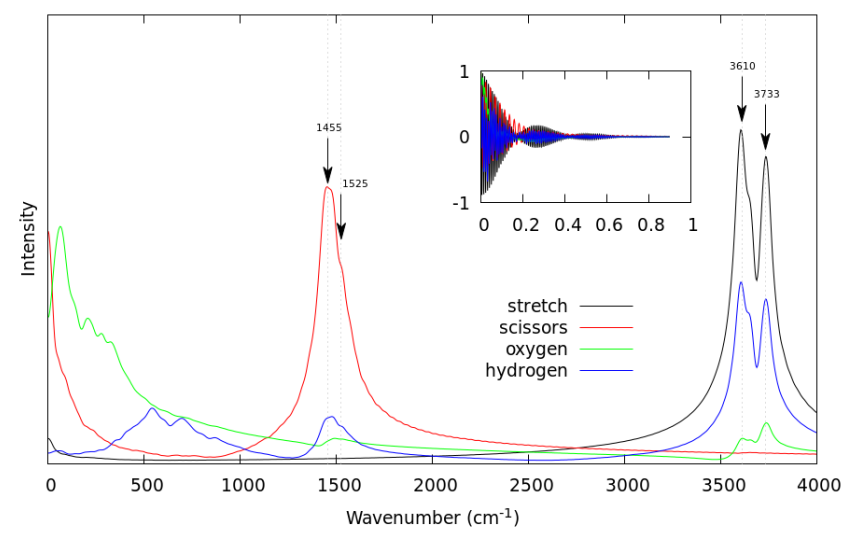

Figure 11. Vibrational analysis of normal modes of $\mathrm{H}_{2} \mathrm{O}$ molecules. The inset graph shows the respective autocorrelation function of each vibrational mode.

\section{Conclusion}

In this work, a new computational package called HICOLM was presented. The purpose is the treatment of atomic-level simulations of condensed systems using theoretical models by low computational cost. Separated into two parts, where the first one is based on the tight-binding method that consists of solution of the generalized eigenvalue equation. Through the tight-binding method, it is possible to obtain the energy levels of electrons in a periodic and homogeneous structure. In order to test the model, results of the graphene band structure containing two carbon atoms were obtained and compared with values in the literature. From a comparison between them, it was noticed that the interatomic distance model, which was proposed in this paper, satisfactorily solves the discrepancies found in the $\sigma$ and $\pi$ bands at points $\Gamma$ and $M$ compared to the traditional model. The second method used in the HICOLM program is the development of classical molecular dynamics which HICOLM program showed widely capable to perform calculations involving complex molecular structures and other carbon-based structures. In order to test the reliability of the program, results involving structure and vibrational analysis of condensed systems were calculated and compared with other ones found in the literature. The analysis of the supercritical argon structure and the vibrational analysis of the liquid water were consistent with results found in the literature, demonstrating that the HICOLM program was able to reproduce satisfactorily the physical behavior of condensed systems. As future work, we intend to expand the Tight-binding model, including the Helmann-Feyman forces in the calculation of intermolecular forces. Thus, a physical analysis at various temperature and pressure ensembles of carbon-based systems can be obtained at thermodynamic equilibrium of tight-binding theory. We also intend to improve the force field of the classical molecular dynamics with the implementation of Ewald sum model for the treatment of Coulomb interactions and the implementation of NoséHoover algorithm for the representation of the NVT and NPT ensembles.

\section{Acknowledgements}

The author thanks Instituto Federal do Paraná for offering all necessary material and financial resources for the development of the project. The author also thanks Prof. Ricardo Wagner Nunes and the Department of Physics of the Instituto de Ciências Exatas of the Universidade Federal de Minas Gerais for their teaching about tight-binding and the density functional theory.

\section{Author contributions}

The paper shows results of a project developed during two years in the field of condensed matter and nanotechnology. The work was entirely developed by the author, without some external collaboration. 


\section{References}

[1] Castro Neto, A. H. et al. The electronic properties of graphene. Reviews of Modern Physics, American Physical Society, v. 81, n. 1, p. 109-162, jan 2009.

[2] MCCANN, E.; KOSHINO, M. The electronic properties of bilayer graphene. Reports on Progress in Physics, IOP Publishing, v. 76, n. 5, p. 056503, may 2013.

[3] KONSCHUH, S.; GMITRA, M.; FABIAN, J. Tightbinding theory of the spin-orbit coupling in graphene. Physical Review B, v. 82, n. 24, p. 245412, 2010.

[4] KONSCHUH, S. Spin-Orbit Coupling Effects From Graphene To Graphite. Tese (Doutorado), 2011.

[5] SAMADIKHAH, K. et al. Continuum-molecular modelling of graphene. Computational Materials Science, Elsevier, v. 53, n. 1, p. 37-43, feb 2012.

[6] CHOI, W. et al. Synthesis of Graphene and Its Applications: A Review. Critical Reviews in Solid State and Materials Sciences, Taylor \& Francis Group, v. 35, n. 1, p. 52-71, feb 2010.

[7] D. Ghuge, A.; R. Shirode, A.; J. Kadam, V. Graphene: A Comprehensive Review. Current Drug Targets, v. 18, n. 6, p. 724-733, mar 2017.

[8] RAJASEKARAN, G.; KUMAR, R.; PARASHAR, A. Tersoff potential with improved accuracy for simulating graphene in molecular dynamics environment. Materials Research Express, v. 3, n. 3, p. 035011, mar 2016.

[9] GRÜNEIS, A. et al. Tight-binding description of the quasiparticle dispersion of graphite and few-layer graphene. Physical Review B, American Physical Society, v. 78, n. 20, p. 205425, nov 2008.

[10] SOOD, A. K. et al. Review of Graphene Technology and Its Applications for Electronic Devices. In: Graphene - New Trends and Developments. [S.1.]: InTech, 2015.

[11] LI, H.; SHI, Y.; LI, L.-J. Synthesis and optoelectronic applications of graphene/transition metal dichalcogenides flatpack assembly. Carbon, Pergamon, v. 127, p. 602-610, feb 2018.

[12] SOLÍS-FERNÁNDEZ, P.; BISSETT, M.; AGO, H. Synthesis, structure and applications of graphene-based 2D heterostructures. Chemical Society Reviews, The Royal Society of Chemistry, v. 46, n. 15, p. 4572-4613, jul 2017.

[13] ABRAHAM, M. J. et al. Gromacs: High performance molecular simulations through multi-level parallelism from laptops to supercomputers. SoftwareX, Elsevier, v. 1-2, p. 1925, sep 2015.

[14] SMITH, W.; YONG, C.; RODGER, P. DL_POLY: Application to molecular simulation. Molecular Simulation, Taylor \& Francis Group, v. 28, n. 5, p. 385-471, may 2002.

[15] PLIMPTON, S. Fast Parallel Algorithms for Short-Range Molecular Dynamics. Journal of Computational Physics, Academic Press, v. 117, n. 1, p. 1-19, mar 1995.
[16] ALLEN, M. P.; TILDESLEY, D. J. Computer Simulation of Liquids. New York, NY, USA: Clarendon Press, 1989.

[17] GUPTA, S. Computing aspects of molecular dynamics simulation. Computer Physics Communications, NorthHolland, v. 70, n. 2, p. 243-270, jun 1992.

[18] EWALD, P. P. Die Berechnung optischer und elektrostatischer Gitterpotentiale. Annalen der Physik, WileyBlackwell, v. 369, n. 3, p. 253-287, jan 1921.

[19] FENNELL, C. J.; GEZELTER, J. D. Is the Ewald summation still necessary? Pairwise alternatives to the accepted standard for long-range electrostatics. The Journal of Chemical Physics, American Institute of Physics, v. 124, n. 23, p. 234104, jun 2006.

[20] WOLF, D. et al. Exact method for the simulation of Coulombic systems by spherically truncated, pairwise $\mathrm{r}-1$ summation. The Journal of Chemical Physics, American Institute of Physics, v. 110, n. 17, p. 8254, apr 1999.

[21] TERSOFF, J. Modeling solid-state chemistry: Interatomic potentials for multicomponent systems. Physical Review B, American Physical Society, v. 39, n. 8, p. 5566-5568, mar 1989.

[22] BERENDSEN, H. J. C. et al. Molecular dynamics with coupling to an external bath. The Journal of Chemical Physics, American Institute of Physics, v. 81, n. 8, p. 3684-3690, oct 1984.

[23] MORÉ et al. The MINPACK Project, in Sources and Development of Mathematical Software. 1984.

[24] MORE, J. J.; GARBOW, B. S.; HILLSTROM, K. E. User Guide for MINPACK-1. Style DeKalb IL, Argonne National Laboratory, 1980.

[25] ANDERSON, E. LAPACK users' guide. [S.1.]: Society for Industrial and Applied Mathematics, 1999. 407 p.

[26] GARCÍA, A. et al. The SIESTA method for ab initio order- N materials simulation. Journal of Physics: Condensed Matter, IOP Publishing, v. 14, n. 11, p. 2745-2779, mar 2002.

[27] WHITE, J. A. Lennard-Jones as a model for argon and test of extended renormalization group calculations. The Journal of Chemical Physics, American Institute of Physics, v. 111, n. 20, p. 9352, nov 1999.

[28] WU, Y.; TEPPER, H. L.; VOTH, G. A. Flexible simple point-charge water model with improved liquid-state properties. The Journal of Chemical Physics, American Institute of Physics, v. 124, n. 2, p. 024503, jan 2006.

[29] SAITO, R.; DRESSELHAUS, G.; DRESSELHAUS, M. S. Physical Properties of Carbon Nanotubes. [S.1.]: PUBLISHED BY IMPERIAL COLLEGE PRESS AND DISTRIBUTED BY WORLD SCIENTIFIC PUBLISHING CO., 2012.

[30] KHAN, A. A. Radial distribution functions of fluid argon. Physical Review, American Physical Society, v. 134, n. 2A, p. A367-A384, apr 1964. 
HICOLM: High-Performance Platform of Large Systems by Using Low Computational Cost Methods

[31] William E. Acree, J. S. C. Phase Transition Enthalpy Measurements of Organic and Organometallic Compounds. In: NIST Chemistry WebBook, NIST Standard Reference Database Number 69, Eds. P.J. Linstrom and W.G. Mallard. Gaithersburg MD: [s.n.], 2019. p. 20899.
[32] TEGELER, C.; SPAN, R.; WAGNER, W. A New Equation of State for Argon Covering the Fluid Region for Temperatures From the Melting Line to $700 \mathrm{~K}$ at Pressures up to 1000 MPa. Journal of Physical and Chemical Reference Data, 1999. 